\title{
A Study of Transform Domain based Image Enhancement Techniques
}

\author{
Gurwinder Kaur \\ M.Tech Scholar \\ Department of Electronics\& Communication \\ Engineering, \\ Beant College of Engineering \& Technology, \\ Gurdaspur
}

\author{
Mandeep Kaur \\ Assistant Professor, \\ Department of Electronics \& Communication \\ Engineering, Beant College of Engineering \& \\ Technology, Gurdaspur
}

\begin{abstract}
An alteration of the low complexity upgrades strategies that are in light of the singular value decomposition (SVD) for saving the mean brightness of a given picture. In spite of the fact that the SVD structured systems upgrade the reduced complexity pictures by scaling its particular worth grid, they may neglect to deliver palatable results for some low difference pictures. The weighted total of solitary lattices of the data picture and its global histogram equalization (GHE) picture is ascertained to get the particular quality framework of the leveled picture. It outflanks the routine picture evening out, for example, GHE and nearby histogram balance (LHE) and in addition the SVD systems that in light of scaling its particular quality both subjectively and quantitatively. The DWT technique can produce better quantitative measurements over the other methods.
\end{abstract}

\section{Keywords}

Image Enhancement, DWT, DCT, SVD

\section{INTRODUCTION}

The primary aim involving image enhancement is usually to improve the caliber of the photograph. The point of snapshot upgrade is usually to enhance your interpretability as well as impression involving data with pictures for human visitors, or to present better facts for some other computerized snapshot handling strategies.

Image enhancement methods may be partitioned straight into two general classifications:

1) Spatial location strategies, that works especially on pixel.

2) Recurrence location strategies, which work with the Fourier change of your picture.

The objective of image enhancement would be to improve the particular impression top quality so the particular processed impression is superior to the key impression to have a particular application or directory ambitions [1]. Lamentably, there is no broad speculation for figuring out what is usually 'good' snapshot improvement in terms of human observation. In the presentation that the idea looks wonderful, it is fantastic! In virtually any case, when snapshot improvement strategies are employed as pre-handling apparatuses for other snapshot preparing devices, then quantitative actions can discover which methods are likely to be proper.

Graphic touch-ups encompasses this functions related to transforming images, if they tend to be digital digital camera pictures, regular photochemical photographs, or designs. Standard analog feeling editing is known as picture retouching or maybe editing designs having any kind of standard fine art channel. Graphic applications, which is usually broadly arranged into vector images writers, raster images writers, as well as 3D modelers, are classified as the major products having which will the end user may modify, increase, as well as alter images.

There are a great deal of impression enhancement strategies are proposed. The distinct worldwide histogram equalization (GHE) approach is probably the most favored comparison enlargement strategies. GHE is often a straightforward as well as effective strategy to alter the particular narrow histogram via distribution as well as stretching out and about this characteristics variety of a good enter impression to get overall comparison enlargement. The neighborhood histogram equalization (LHE) approach however, could boost the entire feeling comparison more effectively, although the particular computational complexity is usually a lot increased automobile by using fully overlapped sub-blocks. 


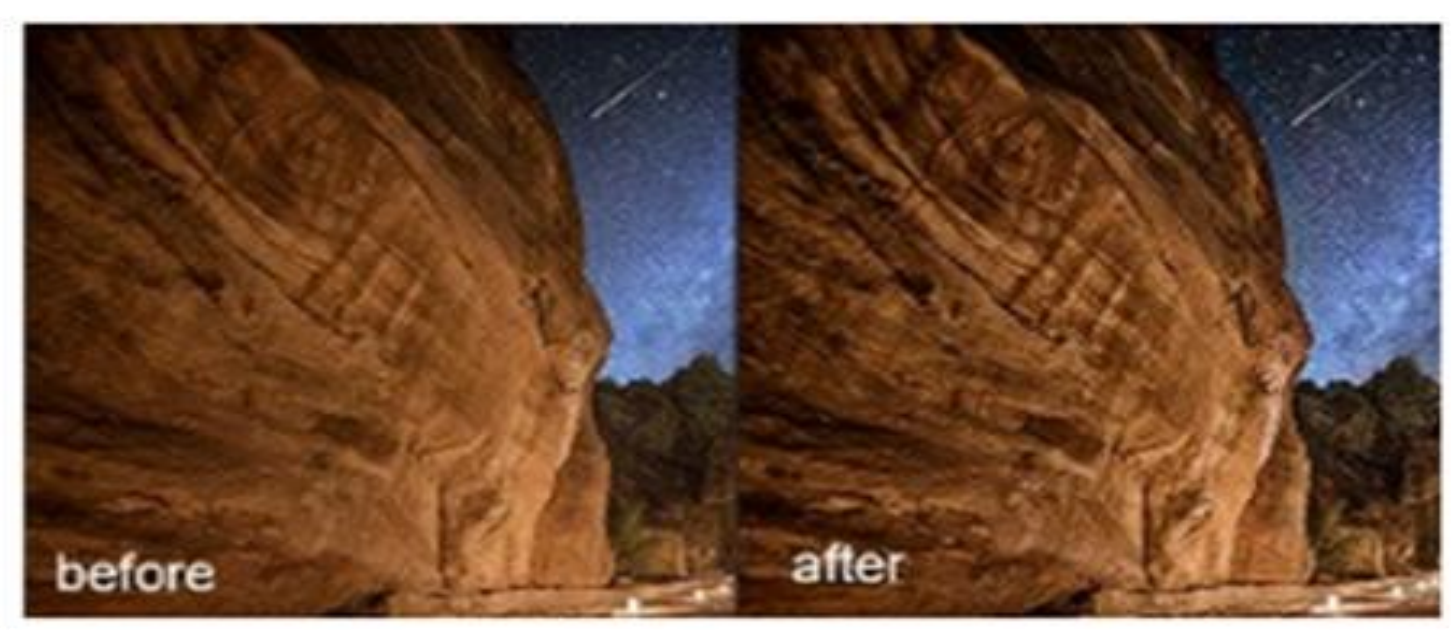

Fig 1: Image Enhancement

\section{IMAGE ENHANCEMENT TECHNIQUES}

A few image improvement techniques have been discussed under.

\subsection{DWT based enhancement:}

Throughout numerical assessment and practical investigation, the individually distinct wavelet change (DWT) is definitely just about any wavelet modify that's the wavelets are usually discretely scrutinized. Likewise along with other wavelet alterations, a important favorable position it's more compared to Fourier alterations is life determination: the item catches both equally recurrence as well as area files. For illustration Haar wavelets as well as Daubechies Wavelets.

2.1.1 Haar wavelets: The primary DWT got its start by Hungarian mathematician Alfréd Haar. With an info spoken to by way of rundown involving numbers, the Haar wavelet change could possibly be considered to fit up files qualities, putting away the distinction and transferring the entirety. This treatment is rehashed recursively, blending the aggregates to provide the using scale, which encourages contrasts and also a last entirety.

2.1.1 Daubechies wavelets: Essentially the most ordinarily used arrangement involving discrete wavelet alterations was detailed because of the Belgian mathematician Ingrid Daubechies throughout 1988. This definition is at light of the utilization involving repeat relations to create continuously far better discrete samplings of your understood new mother wavelet do the job; every dedication is double that of the past scale. In her original report, Daubechies infers several wavelets, the very first of which can be the Haar wavelet. Enthusiasm just for this field possesses blasted from that time forward, and numerous varieties of Daubechies' exclusive wavelets have been developed.

\subsection{DCT based enhancement:}

A individually distinct cosine modify (DCT) communicates a small arrangement involving information focuses where an blend of cosine volumes wavering at diverse frequencies. DCTs usually are imperative to be able to various applications in research and building, from lossy demand of seems (e. whilst gary your gadget dude. MP3) in addition to photos (e. whilst gary your gadget dude. JPEG) to be able to ghastly routines for the numerical arrangement of unfinished differential numerical statements. The using cosine as opposed to sine volumes is discerning for demand, since that being said that a smaller amount cosine capacities are anticipated to rough an incredibly common sign, while regarding differential numerical statements this specific cosines express a selected decision concerning limit circumstances.

Specifically, a DCT is often a Fourier-related change including the discrete Fourier alter (DFT), even so utilizing basically genuine quantities. DCTs are just like DFTs concerning generally double the place, working with genuine information with also symmetry (since this specific Fourier change from the genuine plus capacity will likely be genuine along with even), where inside of a few variations the results and/or make information usually are moved significantly an example. There usually are eight normal DCT types, of of which four usually are normal.

One of the most well-known deviation of individually distinct cosine change will be the sort II DCT, which can be regularly named basically "the DCT" it is opposite, the kind III DCT, will be correspondingly generally called simply "the change DCT" or maybe "the IDCT". Two associated changes are the discrete sine modify (DST), which can be proportionate to some DFT involving genuine in addition to odd volumes, and this changed individually distinct cosine modify (MDCT), which can be in light of the DCT involving covering data.

\subsection{SVD based enhancement:}

With straight polynomial math concepts, the specific quality rot away (SVD) is usually a factorization of the genuine or maybe complex grid. It has numerous helpful applications inside sign preparing and sizes.

Formally, the sole worth decay of the $m \times$ in genuine or maybe complex grid $\mathrm{M}$ is usually a factorization on the structure $\mathrm{M}=\mathrm{U \Sigma V} *$, where $\mathrm{U}$ is usually a $\mathrm{m} \times$ michael genuine or maybe complex unitary lattice, $\Sigma$ is usually a $\mathrm{m} \times$ in rectangular slanting circle with non-negative true numbers around the corner to help corner, in addition to $\mathrm{V} *$ (the conjugate transpose associated with $\mathrm{V}$, or just the transpose associated with $\mathrm{V}$ in case $\mathrm{V}$ is actually genuine) is usually a $\mathrm{n}$ $X$ in genuine or maybe complex unitary construction. The inclining airways $\Sigma \mathrm{i}$, i associated with $\Sigma$ are often known as the sole estimations associated with $\mathrm{M}$. The michael segments associated with $\mathrm{U}$ and also the $\mathrm{n}$ parts of $\mathrm{V}$ are often known as 
the left-solitary vectors in addition to right-particular vectors associated with $\mathrm{M}$, individually.

The sole worth disintegration and also the eigen decomposition are generally firmly associated. Specifically:

1. The actual left-solitary vectors associated with $\mathbf{M}$ are generally eigenvectors associated with $\mathrm{MM} *$.

2. a couple of. The right-particular vectors associated with $\mathrm{M}$ are generally eigenvectors associated with $\mathrm{M} * \mathrm{M}$.

3. The non-zero specific estimations associated with $\mathrm{M}$ (found around the inclining parts of $\Sigma$ ) would be the square bases on the non-zero eigen price.

\section{RELATED WORK}

Atta et al. [1] features proposed an alteration of the low contrast improvement techniques that derive from the singular value decomposition (SVD) intended for preserving the particular mean intensity of any given image While using the proposed technique, the weighted sum of singular matrices from the input image as well as global histogram equalization (GHE) image is calculated to discover the singular worth matrix from the equalized image. Simulation final results show the proposed technique preserves the particular image settings more accurately and enhances it with relatively negligible visual artifacts. It outperforms the standard image equalization like GHE and also local histogram equalization (LHE), as well as the SVD methods that according to scaling the singular worth both qualitatively and also quantitatively. Bhandari et al. [2] explained basic policy for an enhanced and effortless approach intended pertaining to enlargement regarding darkish plus low kind the comparison satellite tv for pc image based on knee operate in addition to gamma static a static correction utilizing underneath the radar wavelet adjust having single value decomposition (DWT-SVD) may be thought out meant for quality improvement associated with credit. In this way may also procedure this hi-res darkish as well as smaller comparison pictures, in addition to provides best enhanced end result using tuning parameter regarding Gamma. The particular arranged criteria overcomes this specific make any difference utilizing lower-leg functionality plus gamma static a static correction. Ghosh et al. [3] discussed the capacity for a new marketing criteria. The actual Cuckoo Find protocol all through tuning the actual impression development features pertaining to peak performance. The actual evaluate has long been carried out in relation to a pair of using the previous optimisation protocol helped improvement, exclusively, Ancestral Algorithms plus Particle Swarm Marketing and sooner improvement methods Histogram Equalization plus Linear Big difference Grow methods. Effects are actually merged with this papers plus a conclusion are actually utilized retaining the actual health and fitness regarding photograph and selection of tips all through much better impression because normal. The results have created the ability regarding Cuckoo investigation protocol all through optimizing the actual improvement attributes .Mathew et 'al. [4] offers a brand new satellite tv for pc photograph decision and as well perfection progress method in line with the discrete wavelet alter (DWT) and as well singular worth decomposition (SVD) is still planned. Satellite tv pictures included in a great deal of apps as an illustration geosciences reports, astronomy, and as well geographical information programs. The particular method additionally quotes the particular fresh worth matrix while using the lowlow striped bass speaker wedding band associated with histogram equalized photograph and alter equally fresh worth matrices to acquire perfection superior graphic. The particular method is actually balanced with conventional photograph equalization techniques for illustration common recurrent histogram equalization and as well community histogram equalization, together with state-of-the-art techniques for illustration perfection safeguarding powerful histogram equalization and as well singular worth equalization. Gupta et al. [5] defined the many existing procedures for that enhancement connected with dark images; it has been observed if the images have certain bright spot, then the item becomes additional bright soon after application of the people techniques. The design has emerged using this obtainable downside. In this short article he provides changed the actual parameter used previous. In feature from the visual demonstration the designed algorithm comes forth as quite simple and well-organized together with keeping attention to the vibrant region that will not to get degraded. The decomposition connected with areas has been done by entropy from the image. Huang et al. [6] explained histogram equalization is often a well-known in addition to successful method of bettering the exact compare associated with photographs but the traditional histogram equalization (HE) plan normally results in excellent compare enlargement, which experts claim reasons an upsetting seem in addition to image artifacts through the highly processed photograph. Very first, this supplied histogram separating module is actually a mixture of this supplied requests many thresholding process and also a excellent the best possible maximum signal-tonoise percentage (PSNR) calculation to split up this histogram using small-scale facet. As the best stage through the offered plan, using this depth change for better component can certainly enhance the picture together with total settings maintenance pertaining to every generated sub-histogram. Fresh results present what sort of offered process other than stores this curve features through the original histogram and improves the exact compare successfully. Xie et al. [7] possesses discussed confront recognition which were representing in addition to recognizing faces depending on subspace discriminant study but also pertaining to individual sample experience acceptance these kinds of solutions are usually usually extraordinary generalizability dilemma due to small biological samples. This particular papers suggests refreshing non-statistics features removal approach depending on fusion including DCT together with neighborhood Gabor binary structure Histogram (LGBPH). Within DCT together with LGBPH, training plan of action is usually preventable to develop this face design, so that the generalize ability dilemma is frequently by natural means eradicated. The sensitive ends in ORL face directories display what sort of worldwide face in addition to neighborhood data could possibly be integrated perfectly soon after degree fusion through worldwide together with neighborhood features, which in turn boost the effectiveness including individual sample experience acceptance. Kim et al. [8] boasts talked about a fresh form a contrast enhancement technique determined by dominant purity amount analyze in addition to adaptive strength transformation relating to out of the way sensing graphics. The idea out and about criteria computes brightness-adaptive strength exchange features with all the low-frequency luminance part inside wavelet spot in addition to switches strength values based on the exchange intent. Although a number of histogram equalization strategies happen to be offered inside literary works, they will usually lower the general graphic high quality by means of showcasing saturation artifacts inside each low- as well as high-intensity destinations. The idea out and about criteria overcomes this trouble with all the adaptive strength exchange 
intent. The new benefits show the fact that planned system improves the general form a contrast as well as visibility affecting local details more than present tactics. The idea out and about approach can efficiently improve any kind of lowcontrast graphics obtained using a satellite tv video camera and they're in addition befitting additional a number of image resolution products which includes purchaser unwanted cameras, photorealistic 3-D renovation systems, as well as computational video cameras. Yonghong et al. [9] has described lighting is attenuated when disseminating with water, the simpleness of images or video lessons captured within water is frequently corrupted to help varying diplomas. In this particular algorithm, a book underwater dim channel springs to approximate the distributing rate, and a highly effective process is usually presented to help estimate the backdrop light inside the underwater optical product. Experimental benefits show which algorithm may well deal with underwater images, particularly pertaining to deep-sea images and the ones captured coming from dirty rich waters. Gupta et al. [10] has referred applications in the Ant Colony Optimization (ACO) to unravel image running problem having a orientation to your new automated enhancement method determined by real-coded particle ant colony can be planned with this paper. The intention of the designed ACO is always to maximize goal fitness criterion to be able to improve the particular contrast as well as detail in the image by means of adapting the particular parameters of a novel extension to your local enlargement method. The probability in the planned method is verified and in contrast to Genetic Algorithms (GAs) as well as Particle Swarm Optimisation (PSO) structured image enlargement technique. The attained results indicate that this planned ACO assure better results with regard to both the particular maximization of the amount of pixels inside the edges as well as the adopted purpose evaluation. Computational time is usually relatively small inside the ACO case when compared to the GA as well as PSO event. Khan et al. [11] viewable another program for denoising regarding pictures even though preserving considerable information for instance blurred lean edges as well as low-contrast good features employing an adaptive threshold in single value decomposition. In this particular different thresholds for that different organised portions in the image are calculated in accordance with local gradient as well as gray amount variance from each pixel position of the portions in the image. An maximum threshold may be estimated through the analysis regarding signal to help noise ratios in the singular importance decomposed images for various thresholds. Demirel et al. [12] proposed a brand new satellite photograph resolution enlargement system using the interpolation inside high-frequency subbands attained simply by under the radar wavelet enhance (DWT) and also the feedback image. The particular predicted quality enlargement technique works by applying DWT that can help decompose the specific feedback image in numerous subbands. And, the high-frequency subband images and also the feedback lowresolution image are interpolated, followed by mixing these images to develop a brand new resolution-enhanced image by utilizing inverse DWT. Braik et al. [13] possesses described applications in the Particle Swarm Marketing (PSO) in order to resolve impression command difficulty using a reference to any novel digital enlargement technique according to real- coded particle swarms might be prepared within this page. The objective within the prepared PSO would be to maximize a target health common so that you can increase the certain distinction and feature in a very artwork by altering the actual details associated with virtually any novel inclusion to your community improvement method. The actual received results reveal how the offered PSO makes far better results concerning both certain maximization associated with the number of pixels through the sides plus the used purpose examination. Computational moment is additionally fairly modest through the PSO scenario compared to the GA function. C.Munteanu et al. [14] possesses discussed photograph enhancement could be the task associated with applying specific transformations to an input image for instance to secure a visually much less noisy productivity image. This paper introduces a fresh automatic photograph enhancement system dependant on an evolutionary marketing process. He supplies a new goal standard intended for enhancement, and tries to locating the finest image good particular common. Due towards high complication in the enhancement common planned, he employ an evolutionary algorithm (EA) to be a global seek plan for the best enhancement. Regarding subjective in addition to objective evaluation, show the particular superiority of the method.

\section{GAPS IN LITERATURE}

Following are the various gaps in earlier work on image enhancement techniques.

1. The effect of noise and edge preservation is ignored.

2. The use of swarm intelligence while enhancing the image is also ignored.

3. The majority of existing research focuses in natural images. The effect of image enhancement on remote sensing image is ignored

\section{PROPOSED METHODOLOGY}

Step 1: Start the algorithm.

Step 2: Then take any remote sensing image.

Step 3: After that apply DWT technique in order to enhance the image.

Step 4: Then select the path which include LL, LH, HL, HH

Step 5: Then apply SVD on it.

Step 6: Apply cuckoo search in order to find optimistic adjustment value.

Step 7: Adjustment of the actual image is done after using optimistic cuckoo search.

Step 8: Then improvement in the LL image is done.

Step 9: Then apply Inverse DWT technique on improved LL.

Step 10: At the end, for the noise removal the trilateral filter is applied on the remote sensing image.

Step 11: Stop the algorithm. 


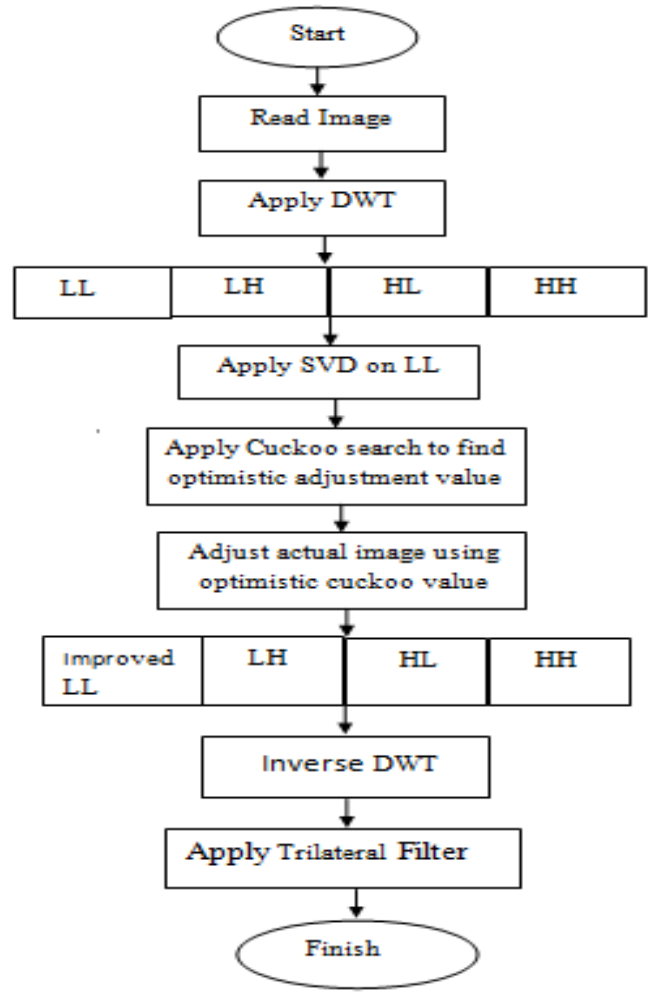

Fig 2: Flowchart of Proposed Algorithm

\section{CONCLUSION}

Additionally, DWT technique can enhance the contrast in the pictures without having presenting unwelcome artifacts that lower aesthetic excellent of an graphic and also make it provide an not naturally made. The use of swarm intelligence while enhancing the image and the majority of existing research focus in natural images. The effect of image enhancement on remote sensing image is also ignored in the previous algorithms. In near future we will utilize the cuckoo search to find optimistic adjustment factor for DWT and SVD and also use trilateral filter in order to preserve edges in more optimistic manner

\section{REFERENCES}

[1] Atta, Randa, and Rabab Farouk Abdel-Kader. "Brightness preserving based on singular value decomposition for image contrast enhancement." OptikInternational Journal for Light and Electron Optics 126, no. $7:$ 799-803, 2015.

[2] Bhandari, A. K., Anil Kumar, G. K. Singh, and Vivek Soni. "Dark satellite image enhancement using knee transfer function and gamma correction based on DWTSVD." Multidimensional Systems and Signal Processing : 1-24, 2015.

[3] Ghosh, Soham, Sourya Roy, Utkarsh Kumar, and Arijit Mallick. "Gray Level Image Enhancement Using Cuckoo Search Algorithm." In Advances in Signal Processing and Intelligent Recognition Systems, pp. 275-286. Springer International Publishing, 2014

[4] Mathew, Ammu Anna, and S. Kamatchi. "Brightness and Resolution Enhancement of Satellite Images using SVD and DWT." International Journal of Engineering Trends and Technology 4, no. $4: 712-718,2013$.

[5] Gupta, Nidhi, and Rajib Jha. "Enhancement of High Dynamic Range Dark Images Using Internal Noise in DWT Domain." In Intelligent Interactive Technologies and Multimedia, pp. 66-74. Springer Berlin Heidelberg, 2013.

[6] Huang, Shih-Chia and Chien-Hui Yeh. "Image contrast enhancement for preserving mean brightness without losing image features." Engineering Applications of Artificial Intelligence 26, no. 5 : 1487-1492, 2013.

[7] Xie, Zhihua. "Single sample face recognition based on dct and local Gabor binary pattern histogram." In Intelligent Computing Theories, pp. 435-442. Springer Berlin Heidelberg, 2013.

[8] Lee, Edward, Sungho Kim, Wei Kang, Daeban Seo, and Jamie Paik. "Contrast enhancement using dominant brightness level analysis and adaptive intensity transformation for remote sensing images." Geoscience and Remote Sensing Letters, IEEE 10, no. 1 : 62-66, 2013.

[9] Wen, Haocheng, Yonghong Tian, Tiejun Huang, and Wen Gao "Single underwater image enhancement with a new optical model." In Circuits and Systems, IEEE International Symposium on, pp. 753-756, 2013.

[10] Gupta, Kanika, and Akshu Gupta. "Image enhancement using ant colony optimization." IOSR J. VLSI Signal Process $1: 38,2012$.

[11] Khan, Nafis Uddin, K. V. Arya, and Manisha Pattanaik. "A New Adaptive Thresholding in SVD for Efficient Image De-noising." In Proceedings of the International Conference on Soft Computing for Problem Solving, pp. 659-670. Springer India, 2012.

[12] Demirel, Hasan, and Gholamreza Anbarjafari. "Discrete wavelet transform-based satellite image resolution enhancement." Geoscience and Remote Sensing, IEEE Transactions on 49, no. 6: 1997-2004 2011.

[13] Braik, Malik, Alaa F. Sheta, and Aladdin Ayesh. "Image Enhancement Using Particle Swarm Optimization." In World congress on engineering, vol. 1, pp. 978-988. 2007.

[14] Munteanu, Cristian, and Agostinho Rosa. "Gray-scale image enhancement as an automatic process driven by evolution." Systems, Man, and Cybernetics, Part B: Cybernetics, IEEE Transactions on 34, no. 2 : 12921298, 2004. 